\title{
BMJ Open Deprescribing intervention activities mapped to guiding principles for use in general practice: a scoping review
}

\author{
Amy Coe (D) , ' Catherine Kaylor-Hughes (D) , Susan Fletcher (D) , \\ Elizabeth Murray (D) ,2 Jane Gunn (D) ${ }^{1}$
}

To cite: Coe A, Kaylor-Hughes C, Fletcher S, et al. Deprescribing intervention activities mapped to guiding principles for use in general practice: a scoping review. BMJ Open 2021;11:e052547. doi:10.1136/ bmjopen-2021-052547

- Prepublication history and additional supplemental material for this paper are available online. To view these files, please visit the journal online (http://dx.doi.org/10.1136/ bmjopen-2021-052547)

Received 20 April 2021 Accepted 20 August 2021

Check for updates

(C) Author(s) (or their employer(s)) 2021. Re-use permitted under CC BY-NC. No commercial re-use. See rights and permissions. Published by BMJ.

${ }^{1}$ Department of General Practice, The University of Melbourne, Melbourne, Victoria, Australia ${ }^{2}$ Primary Care and Population Health, University College London, London, UK

Correspondence to

Ms Amy Coe;

amy.coe@unimelb.edu.au

\section{ABSTRACT}

Objective To identify and characterise activities for deprescribing used in general practice and to map the identified activities to pioneering principles of deprescribing.

Setting Primary care.

Data sources Medline, EMBASE (Ovid), CINAHL, Australian New Zealand Clinical Trials Registry (ANZCTR), Clinicaltrials.gov, ISRCTN registry, OpenGrey, Annals of Family Medicine, BMC Family Practice, Family Practice and British Journal of General Practice (BJGP) from inception to the end of June 2021.

Study selection Included studies were original research (randomised controlled trial, quasi-experimental, cohort study, qualitative and case studies), protocol papers and protocol registrations.

Data extraction Screening and data extraction was completed by one reviewer; $10 \%$ of the studies were independently reviewed by a second reviewer. Coding of full-text articles in NVivo was conducted and mapped to five deprescribing principles.

Results Fifty studies were included. The most frequently used activities were identification of appropriate patients for deprescribing (76\%), patient education (50\%), general practitioners (GP) education (48\%), and development and use of a tapering schedule (38\%). Six activities did not align with the five deprescribing principles. As such, two principles (engage practice staff in education and appropriate identification of patients, and provide feedback to staff about deprescribing occurrences within the practice) were added.

Conclusion Activities and guiding principles for deprescribing should be paired together to provide an accessible and comprehensive guide to deprescribing by GPs. The addition of two principles suggests that practice staff and practice management teams may play an instrumental role in sustaining deprescribing processes within clinical practice. Future research is required to determine the most of effective activities to use within each principle and by whom

\section{INTRODUCTION}

The WHO estimates that half of all medicines prescribed worldwide are done so inappropriately. 'Inappropriate medication use (IMU)' can occur when medications are prescribed and taken despite there being no clinical

\section{Strengths and limitations of this study}

First study to investigate deprescribing activities in general practice.

- First study to map deprescribing activities to guiding principles.

- This study used a robust, up-to-date and comprehensive search strategy.

- Critical appraisal of studies was not conducted.

- Effectiveness and outcomes of the identified activities were not examined.

benefit or the risk of taking a medication outweighs the benefit. ${ }^{2}$ IMU is often linked with polypharmacy where patients with multiple health issues are prescribed multiple medications, placing them at increased risk of adverse reactions and interactions. ${ }^{3}$ It is estimated that $20 \%-30 \%$ of the general population experience harmful events due to IMU and polypharmacy resulting in hospitalisation and increased risk of mortality. ${ }^{45}$ Deprescribing ('the planned and supervised process of dose reduction or stopping unnecessary or potentially harmful medication' $)^{6}$ is a recommended component of best practice prescribing which can address the issues of IMU and polypharmacy. Both prescribing and deprescribing require skilful and careful clinical judgement to balance the risks and benefits of medicines, minimising potential harms and improving patient health outcomes. $^{7}$ General practitioners (GPs) prescribe the majority of medications ${ }^{8}$ and are well placed to conduct the majority of deprescribing. However, deprescribing is not routinely occurring in clinical practice. ${ }^{79}$

Evidence suggests that patients are willing to cease unnecessary medications but require empowerment and engagement from their GP to do so and are likely to leave it to their GP to initiate the deprescribing conversation. ${ }^{10}$ However, research has identified a number of barriers to this occurring, including 
appointment time constraints, lack of good quality guidelines, ${ }^{9}$ clinical inertia ${ }^{11}$ and not knowing when to deprescribe. ${ }^{12}$ When asked about what would assist with their deprescribing role, GPs express a desire to have support and work in collaboration with other healthcare professionals, ${ }^{9}$ have ready access to non-pharmacological options and resources, and decision-making systems and tools ${ }^{13}$ to enable them to regularly and confidently conduct deprescribing.

Activities to support GPs to deprescribe have been investigated, although only one systematic review has focused on deprescribing by GPs in primary care. This review by Dills et $a l^{14}$ found three effective activities for successful deprescribing: (1) pharmacist-physician collaboration for conducting medication reviews; (2) giving clinicians intensive education about deprescribing; and (3) providing individual patients with information about chronic disease management and IMU (eg, pharmacological and lifestyle advice and alternative options for treatment). ${ }^{14}$ Most of the included studies were set in long-term care, assisted living and outpatients, which are commonly considered to fall outside the definition of primary care. Further, only 6 of the 58 studies were conducted in general practice. Although GPs do practise in these settings, the effectiveness of the identified activities may not be generalisable to GPs practising specifically within the general practice setting.

Isenor et $a l^{15}$ recently explored deprescribing activities in primary care, which included pharmacy, general practice and allied health. Results of this scoping review revealed that checklists, algorithms, leaflets, patient finder tools, goal setting tools, and prompts or cues in the form of reports, letters, posters or electronic medical record alerts were most frequently used to support deprescribing. These activities were often used in conjunction to form interventions to change GP and patient behaviour. This suggests that deprescribing interventions are multifaceted and employ a variety of techniques to encourage deprescribing at the patient, clinician and systems levels. ${ }^{13}$ Results showed that GPs were the most targeted healthcare professional for intervention, with pharmacists most commonly conducting the deprescribing process. Although pharmacists may play an important role in deprescribing, what activities GPs are using in practice remain unclear.

It is also important to consider how deprescribing activities are being used in practice as this process is essential for successful deprescribing. ${ }^{16}$ Research indicates that how deprescribing activities are delivered has previously been under-reported in deprescribing trials, making it challenging to apply deprescribing evidence into clinical practice. ${ }^{17}$ In the absence of a gold standard deprescribing process, Woodward's five principles of deprescribing offer a strong framework and are core to the deprescribing process. ${ }^{18}$ The five principles of deprescribing consist of (1) review all current medications; (2) identify medications to be targeted for cessation; (3) plan a deprescribing regimen; (4) plan in partnership with patient and carers; and (5) frequent review and support (see figure 1). ${ }^{6}$ Woodward's principles were the first deprescribing guiding principles described in the literature and state that deprescribing should be a collaboration between the prescriber and patient, with subsequent adaptations placing an even greater emphasis on the importance of patient-centred care. ${ }^{18} 19$ The principles were developed with corresponding deprescribing activities; however, whether deprescribing interventions are following these recommendations is not known.

To date, research has focused on deprescribing activities or adapting deprescribing principles, independently, rather than consolidating the two for use in practice. Further, to our knowledge, no reviews have looked specifically at deprescribing activities and principles in general practice. Examining activities and principles together may help to identify areas of the deprescribing process that require attention and provide a comprehensive and accessible knowledge base for GPs, to support and inform their decision-making around deprescribing. As scoping reviews have become a popular, rigorous and transparent method for providing in-depth and comprehensive coverage of the literature, ${ }^{18}$ we conducted a scoping review to provide an up-to-date and inclusive look at deprescribing activities in general practice and map them to a well-known set of deprescribing principles. Specifically, we aimed to (1) provide a summary of the deprescribing literature across all medical conditions presenting to general practice; (2) map the activities to Woodward's five principles of deprescribing; and (3) identify any key deprescribing activities being tested in general practice interventions.

\section{METHOD}

\section{Search strategy}

Methodology was decided on in April 2020 via discussion between authors. A research librarian at the University
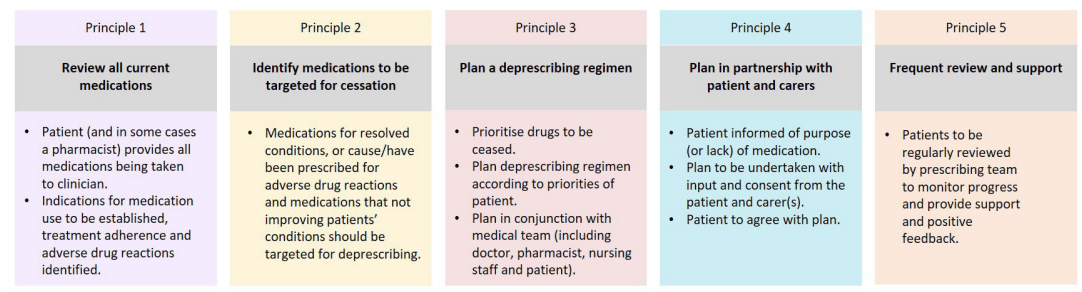

Figure 1 Woodward's five principles for deprescribing. ${ }^{6}$ 


\begin{tabular}{|c|c|}
\hline Concept & Keywords \\
\hline Primary care & $\begin{array}{l}\text { general practice or primary health care or } \\
\text { general practice or general practitioner or } \\
\text { primary care professional }\end{array}$ \\
\hline Deprescribing & $\begin{array}{l}\text { discontinu* or reduc* or terminat }{ }^{\star} \text { or taper } \\
\text { or cease or cessation or stop taking or stop } \\
\text { using or deprescrib* or deprescrip }\end{array}$ \\
\hline Medication & $\begin{array}{l}\text { medic }^{\star} \text { or drug } \\
\text { treatment }{ }^{\star}\end{array}$ \\
\hline
\end{tabular}

of Melbourne was consulted to develop search terms and methods. Studies were identified by searching electronic databases Medline, EMBASE (Ovid), CINAHL, Australian New Zealand Clinical Trials Registry (ANZCTR), Clinicaltrials.gov, ISRCTN registry and OpenGrey from inception to the end of June 2021. Handsearches of four primary care journals (Annals of Family Medicine, BMC Family Practice, Family Practice and Journal of General Practice (BJGP)) were conducted using the search function provided by the journal websites. See table 1 for key concepts for searching and online supplemental appendix A for full search activity.

\section{Participants}

Studies that focused on adults attending general practice and/or healthcare professionals in general practice were included, regardless of the primary diagnosis, type of healthcare professional delivering care, country in which study took place or year published. Studies were excluded if they were not conducted with human participants. Studies focusing on participants younger than 18 years of age were also excluded due to the unique nature of deprescribing medications in paediatric populations (for example see Begum and Tomlin ${ }^{20}$ ).

\section{Setting}

Studies were eligible for inclusion if they were set in general practice (ie, participants were recruited from, or deprescribing was conducted in, a general practice clinic), and if the medication being deprescribed was one that was taken orally. Studies where patients were recruited from general practice but the majority of the deprescribing process was conducted in the patients' home or other setting were excluded. Studies that did not describe the activities of the deprescribing intervention and if they focused on prescribing/deprescribing prevalence or adherence/non-adherence were excluded.

\section{Types of studies}

Studies were included if they were original research (randomised controlled trial (RCT), quasi-experimental, cohort study, qualitative and case studies). Systematic reviews and meta-analyses were included for handsearching purposes. Protocol papers and protocol registrations were included as they describe interventions that are being prepared for trial. Where the full protocol paper was available, this took precedence over the protocol registration record. Articles were excluded if they were non-empirical research (editorials, guidelines/guideline development, commentaries, opinions, letters, factsheets, clinical education activities). Conference abstracts were also excluded as they often lack in-depth intervention descriptions. Studies were excluded if a deprescribing intervention was not the focus. Study quality was not formally assessed and was not an inclusion criteria as this is not a requirement of scoping reviews. ${ }^{21}$

\section{Study selection}

One reviewer (AC) reviewed all titles and abstracts for eligibility, and $10 \%$ of titles and abstracts were separately reviewed by a second reviewer (CK-H) for agreement. The eligible full text articles were downloaded into the COVIDENCE web-based screening and data extraction tool. ${ }^{20}$ Two reviewers (AC and CK-H) independently evaluated $10 \%$ of the full-text articles to decide if they meet the inclusion/exclusion criteria. Five articles required discussion between the two reviewers to resolve disagreement about inclusion. Consultation with a third reviewer was not needed as agreement between the reviewing authors was reached. AC then reviewed all remaining full-text articles for inclusion. A data extraction form was developed to gather the following information for all included studies: author(s), country and year of publication, study type, population, setting, methodology, primary medical condition, medication to be deprescribed, comparator information and study results. To extract information about the deprescribing activities used in the interventions, full-text articles were uploaded into $\mathrm{NVivo}^{22}$ and coded by AC.

\section{Categorisation of the results}

Initial reason for medication prescription targeted for deprescribing was categorised by the International Classification of Diseases 11th Revision (ICD-11) ${ }^{23}$ where possible. To create order for the complex material found in the included studies, intervention activities were categorised into 'who', 'what', 'how' and 'where'. Specifically, 'what' activities were mapped to the five principles as these are the activities that most pertain to the deprescribing process. For patients, GPs, allied health and eTools that were categorised under 'who' were further classified as 'lead' or 'support'. A lead role was assigned if they initiated and oversaw the deprescribing process. A support role indicated they were not the initiator or overseer, but were involved in the deprescribing process. eTools were categorised under 'who' as they were used in place of a person to conduct deprescribing activities. Any activities not mapped to the five principles were grouped together to determine if they contained common traits to form additional principles. Additional principles were named for when the mapped activities took place within the original five principles. 


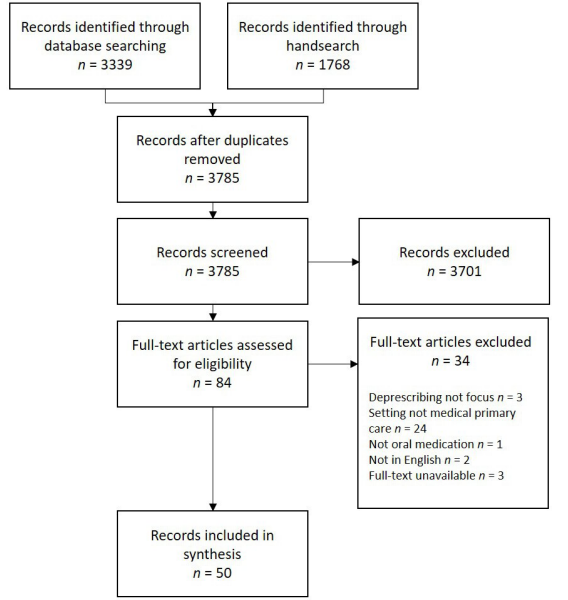

Figure 2 PRISMA (Preferred Reporting Items for Systematic Reviews and Meta-Analyses) flow diagram showing results of search and process of selecting articles for deprescribing scoping review.

\section{Patient and public involvement}

Patients and/or the public were not involved in the design, conduct, reporting or dissemination plans of this research.

\section{RESULTS}

The search yielded a total of 5107 articles, 3785 after duplicates were removed. Review of titles and abstracts led to the retrieval of 84 full-text articles for assessment. Of these, 50 empirical research studies were included (see online supplemental appendix B for individual study characteristics). Figure 2 shows the flow of articles through the search and eligibility screening process.

Included articles were published between 1983 and 2021, with an increase in publication rates in the last 5 years (table 2). Research was primarily conducted in the UK $(\mathrm{n}=9(18 \%))$, The Netherlands $(\mathrm{n}=7(14 \%))$ and the USA $(\mathrm{n}=7(14 \%))$. Twenty studies specifically targeted older patients (aged 60 years and older). ${ }^{24-43}$

Most studies were randomised controlled trials (RCTs) $(\mathrm{n}=31(62 \%))$ and aimed to reduce polypharmacy $(\mathrm{n}=16$ $(32 \%))$ and benzodiazepine use $(\mathrm{n}=14(28 \%))$. Definitions of polypharmacy varied between studies, ranging from $\geq 2$ medications ${ }^{24}$ to $\geq 15$ medications. ${ }^{25}$ Two studies did not specify what the target medication was initially prescribed for-one focused on falls prevention, the other on long-term use. ${ }^{2644}$ In a third of the studies, the target medication was initially prescribed for the treatment of mental illness. The most common reason for deprescribing was medications deemed as IMU ( $\mathrm{n}=26$ $(52 \%))$. Some studies specifically targeted a subset of IMU (eg, long-term use) which is presented as an individual reason for deprescribing.

\section{Activities and principles of deprescribing}

Deprescribing activities and principles were applied across populations, diagnoses and medication types.
Table 2 Characteristics of publications on deprescribing activities

\begin{tabular}{|lrl|}
\hline Characteristics & $\mathbf{N}=\mathbf{5 0}$ & \% of $\mathbf{5 0}$ \\
\hline Type of article & & \\
\hline Randomised controlled trial & \\
\hline Quasi-experimental design & 31 & 62 \\
\hline Cohort studies & 11 & 22 \\
\hline Feasibility studies & 4 & 8 \\
\hline Case-controlled studies & 3 & 6 \\
\hline Country of origin & 1 & 2 \\
\hline UK & & \\
\hline The Netherlands & 9 & 18 \\
\hline USA & 7 & 14 \\
\hline Spain & 7 & 14 \\
\hline Australia & 6 & 12 \\
\hline Canada & 4 & 8 \\
\hline Ireland & 4 & 8 \\
\hline New Zealand & 4 & 8 \\
\hline France & 2 & 4 \\
\hline Portugal & 2 & 4 \\
\hline Switzerland & 1 & 2 \\
\hline Germany & 1 & 2 \\
\hline Scotland & 1 & 2 \\
\hline Multiple locations & 1 & 2 \\
\hline Year of publiation & 1 & 2 \\
\hline
\end{tabular}

Year of publication

\begin{tabular}{lrr}
$<1999$ & 4 & 8 \\
$2000-2005$ & 5 & 10 \\
$2006-2010$ & 4 & 8 \\
$2011-2015$ & 7 & 14 \\
$2016-2021$ & 31 & 60 \\
\hline
\end{tabular}

ICD-11 category

\begin{tabular}{|rrr}
\hline Mental illnesses & 17 & 33 \\
\hline Digestive illnesses & 5 & 10 \\
\hline Multimorbidity & 4 & 8 \\
\hline Nervous system & 2 & 4 \\
\hline General symptoms & 3 & 6 \\
\hline Circulatory & 2 & 4 \\
\hline Sleep-wake disorders & 2 & 4 \\
\hline Infectious & 1 & 2 \\
\hline Other & 4 & 8 \\
\hline Initial reason for prescription not givent & 10 & 20 \\
\hline Specific medication targeted for & & \\
deprescription & & \\
\hline Polypharmacy & 16 & 32 \\
\hline Benzodiazepines & 14 & 28 \\
\hline Antidepressants & 6 & 12 \\
\hline Proton pump inhibitors & 5 & 10 \\
\hline & & Continued \\
\hline
\end{tabular}

Continued 


\begin{tabular}{lcc}
\hline Table 2 Continued & & \\
\hline Characteristics & N=50 & \% of 50 \\
\hline Opioids & 3 & 6 \\
Antihypertensives & 2 & 4 \\
Psychotropics & 1 & 2 \\
Antibiotics & 1 & 2 \\
Anticholinergics & 1 & 2 \\
Mirabegon (urinary incontinence) & 1 & 2 \\
Reason for deprescription & & \\
Inappropriate medication use & 27 & 53 \\
Long-term use & 19 & 37 \\
Adverse side effects & 4 & 8 \\
Exploration of alternative treatment & 1 & 2 \\
\hline
\end{tabular}

NB.

${ }^{*}$ Of the included RCTs, 10 were protocol papers and 11 were protocol registrations.

†These studies targeted polypharmacy, therefore initial reason for the prescription of multiple medications was not specified.

ICD, International Classification of Diseases; RCTs, randomised controlled trials.

Overall, 17 activities were identified and were mapped to seven principles. Six activities did not fit within the original five principles, therefore two additional principles were created: principle 0: engage practice staff in education and appropriate identification of patients and principle 6: provide feedback to staff about deprescribing occurrences within the practice. Principle 0 included five activities which occurred prior to activities mapped to Woodward's five principles. Principle 6 included one activity which occurred after the five principles.
Unsurprisingly, GPs and patients were heavily involved in the deprescribing process. Activities of deprescribing were administered in several different ways including medical records and documents. Deprescribing activities were mainly carried out in the general practice clinic. Figure 3 shows the deprescribing activities mapped to the corresponding principle including who is involved in the deprescribing process, how activities and principles might be administered and where they take place. Figure 4 presents Woodward's five principles with the addition of principle 0 and principle 6 .

\section{Principle 1: review of all current medications}

A review of all medications was conducted in 11 studies $^{24} 25273033364041434546$ and was the only activity mapped to principle 1. GPs most commonly lead this activity, ${ }^{2425} 27364041434546$ with pharmacists ${ }^{30}$ and eTools ${ }^{33}$ also given a lead role.

\section{Principle 2: identify medications to be targeted for cessation} Identification of medications for cessation was conducted in 15 studies. ${ }^{24} 25$ 30-33 36 40-43 46-49 This was led mostly by GPs $(\mathrm{n}=13),{ }^{25} 3132363840-4246-50$ with a pharmacist ${ }^{30}$ and an eTool $^{33}$ leading two further studies. In one study, leadership of identifying medications was shared by a GP, practice nurse or pharmacist. ${ }^{47}$ Identifying the medications for cessation was were often supported by algorithms $(n=9)$ that used information from the review of medications in principle 1 and made recommendations for which medications to target for deprescribing. ${ }^{30-32} 363841434950$ Four studies incorporated the algorithm in an eTool. ${ }^{32} 363841$

\section{Principle 3: plan a deprescribing regimen}

Documented plans for deprescribing were made in five studies. ${ }^{2529325152}$ A variety of healthcare professionals were
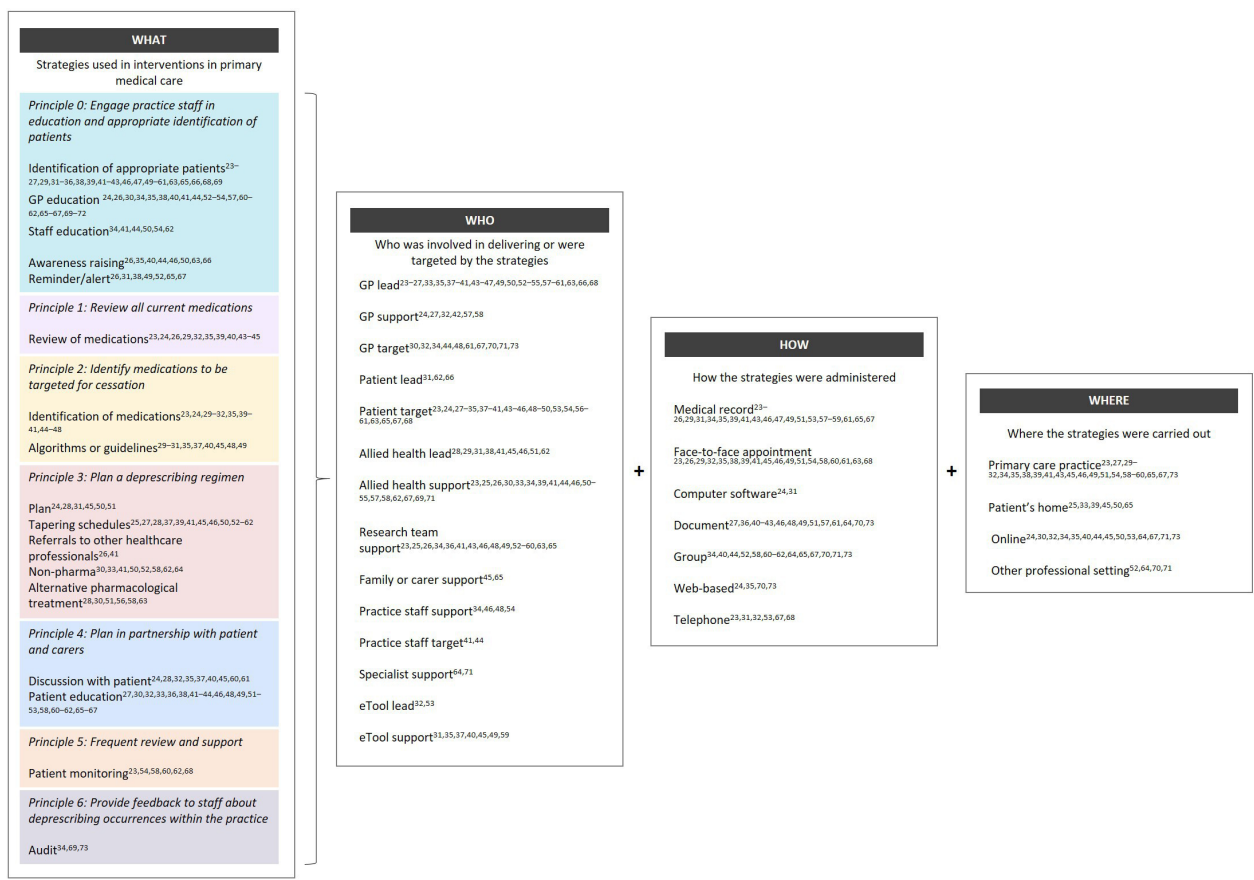

Figure 3 Deprescribing activities mapped to corresponding principles. GP, general practitioner. 


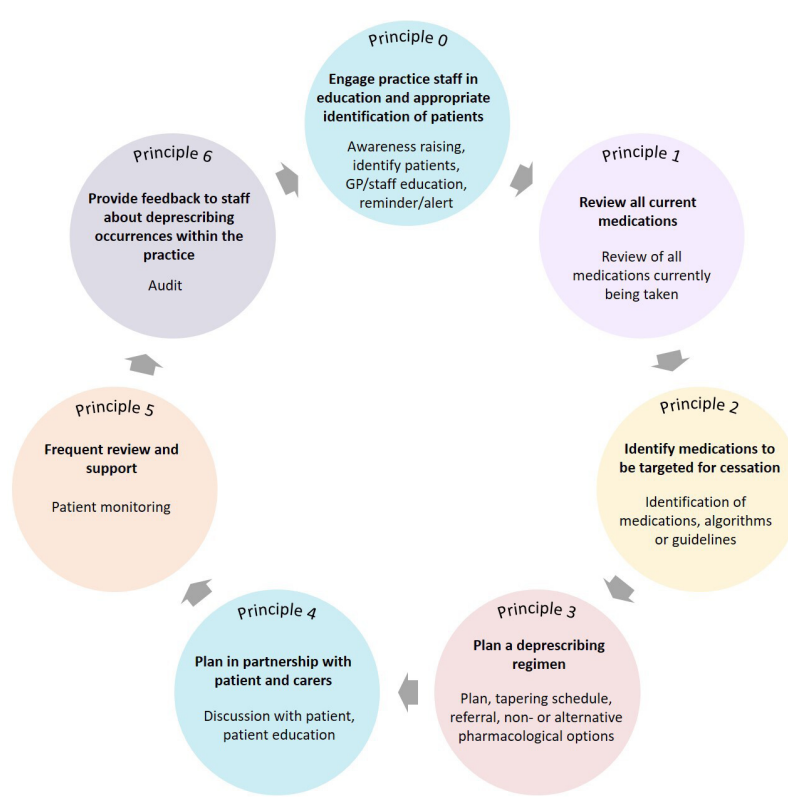

Figure 4 Adapted deprescribing principles based on Woodward's five principles of deprescribing. GP, general practitioner.

involved in this process including pharmacist leads, ${ }^{29} 32$ an eTool, ${ }^{25}$ a nurse ${ }^{52}$ and a GP. ${ }^{51}$ Tapering schedules were widely used $(n=19)^{262829384042475153-63}$ and were delivered by GPs ${ }^{28} 384047515562$ and pharmacists. ${ }^{29}$ A pharmacist, GP and nurse were responsible for tapering schedules in one study. ${ }^{63}$ eTools were used in two studies. ${ }^{540}$ In 10 studies researchers developed and disseminated the tapering schedule to participants. ${ }^{26} 42505356-596164$ Referrals to other healthcare professionals, ${ }^{27} 42$ non-pharmacological options $^{31} 34425153596365$ and alternative pharmacological options 293152575964 were also mapped under principle 3 . These activities were used to support patients to deprescribe after receiving a tapering or deprescribing plan. Non-pharmacological options included guided mindfulness-based cognitive therapy $^{51}$ and exercise programmes. $^{63}$

\section{Principle 4: plan in partnership with patient and carers}

Patients were included in the deprescribing discussions in eight studies. ${ }^{2529} 333638416162$ A deprescribing decision aid was used as a tool in one study to facilitate the deprescribing discussion. ${ }^{51}$ Carers were included in one study, although they were not involved in the deprescribing discussion. GPs conducted discussions with patients in six studies ${ }^{25} 3031386162$ and were aided by an eTool in three studies $^{33} 3641$ and a pharmacist in one study. ${ }^{29}$

Patient education was also a commonly occurring strategy $(n=25) .^{28} 31333437394244-47495052-545961-6366-69$ Education consisted of advice and information about when and how to reduce medications delivered in a variety of ways including receiving a letter in the mail, ${ }^{29}$ advice from their GP. ${ }^{28}$ One study used internet modules ${ }^{54}$ to extend this information by providing material on dealing with withdrawal symptoms and relapse, overcoming fear of stopping and staying well.

\section{Principle 5: frequent review and support}

Six studies reported five different approaches to monitoring patients after the deprescribing process was initiated. ${ }^{245559616369}$ Monitoring involved follow-up telephone appointments $^{24}{ }^{69}$; follow-up in-person appointments which focused on the provision of positive reinforcement, ${ }^{61}$ tracking of physiological responses to deprescribing (eg, blood pressure and cholesterol checks) $)^{55} 63$ and completion of case reports. ${ }^{59}$ Follow-up timeframes ranged from 2 days ${ }^{69}$ to 6 months ${ }^{55}$ following enrolment in the study. Patients attended up to four ${ }^{59} 61$ follow-up visits over the course of the intervention. Three studies did not specify when follow-up visits occurred and four did not specify the number of follow-visits included in the intervention.

\section{Principle 0: engage practice staff in education and appropriate identification of patients and Principle 6: provide feedback to staff about deprescribing occurrences within the practice}

Five activities were mapped to 'Principle 0: engage practice staff in education and appropriate identification of patients', including the most frequently occurring activities found across all of the studies: identifying patients and GP education. Awareness raising of deprescribing among healthcare professionals and reminders and alerts for clinicians were also mapped to this principle. Each of these activities appear to be tasks that GPs, other healthcare professionals and general practice clinics should complete before patient appointments and medication management occurs. Identification of appropriate patients who were eligible for deprescribing occurred in most studies $(\mathrm{n}=38){ }^{24-28} 3032-37394042-45474850-626466676970$ Although this may have occurred as study participant selection, it was included as an activity of deprescribing as GPs need to know which patients to initiate the deprescribing discussions with. GP education occurred in almost half of the studies $(\mathrm{n}=24)^{25} 273135363941-434653-55$ 58 61-63 66-68 70-73 and was conducted prior to any patient contact and therefore before deprescribing started. GP education was delivered in a variety of ways including workshops, ${ }^{27}$ training videos $^{2543}$ and as part of GP medical training. ${ }^{65} 72$ Practice staff education occurred less frequently ${ }^{35} 424346515563$ and typically involved staff being invited to attend the education provided to GPs, rather than delivery of separate or tailored training. Awareness raising was achieved in general practices through practice recruitment and training in study protocols, practice sign-up and participant recruitment $(\mathrm{n}=9) .{ }^{27} 3641434647516467$ Eight studies $^{27} 32394350536668$ used reminders or alerts mostly via patient medical records to notify GPs that a patient with an upcoming appointment required a medication review.

The only activity mapped to principle 6 was the auditing of deprescribing occurrences in practice researchers in three studies. ${ }^{3570} 73$ One study provided practices with comparisons of quality of care against agreed-on standards of practice in the form of a report at unspecified intervals. ${ }^{35}$ Two studies used monthly reports given to 
GPs with one including intervention monitoring information $^{70}$ and the other providing information regarding GP benzodiazepine prescriptions. ${ }^{73}$

\section{Key deprescribing activities}

Four deprescribing activities were the most commonly used in the 50 reviewed studies: (1) $76 \%$ of studies used identification of appropriate patients; (2) $50 \%$ used patient education; (3) $48 \%$ used GP education; and (4) $38 \%$ used a tapering schedule. Identification of these key activities may guide the development of future deprescribing interventions in general practice as well as provide a quick reference for GPs of deprescribing activities in clinical practice.

\section{DISCUSSION}

Deprescribing is critical to addressing the well-recognised problem of IMU, but is currently underperformed in general practice. In looking to assist GPs to engage in deprescribing this, scoping review amalgamated deprescribing activities being used in general practice with pioneering principles of deprescribing. This may provide GPs with a comprehensive and accessible knowledge base for when to use deprescribing activities principles in clinical practice.

Two principles were added to Woodward's original five principles of deprescribing ${ }^{4}$ addressing an area of concern in the literature regarding the lack of GP-initiated deprescribing. Principle 0 encompassed activities aimed at helping GPs to initiate the deprescribing conversation. Auditing activities mapped to principle 6 may also complement principle 0 as auditing information allows staff to improve professional practice. ${ }^{74}$ Providing GPs with information about their own deprescribing practices may improve initiation of the deprescribing process.

The most frequently occurring activities were identifying appropriate patients for deprescribing, patient and GP education and using tapering schedules. Identifying which patients require deprescribing was classified as a deprescribing activity in the current study. Although this activity was used as part of study eligibility, this may be important for the initiation of the deprescribing process for GPs and warrants further testing.

Our findings are consistent with previous literature that has found heterogeneity in the deprescribing process. In particular, the current review adds support for GP and patient education being critical components of the deprescribing process as suggested by Dills et al. ${ }^{14}$ However, identifying appropriate patients for deprescribing has not previously been specified as deprescribing activity to be used in practice and highlights a current gap in the literature.

Focusing on deprescribing conducted solely in general practice yielded different findings from previous literature. We found that $32 \%$ of the included studies focused on polypharmacy compared other reviews that included a wide array of primary care settings (eg, $65 \%$ of studies in the scoping review by Isenor et al). ${ }^{15}$ Traditionally, polypharmacy is an issue for patients aged 65 years and older. As general practice is most commonly attended by adults aged $20-64,{ }^{74}$ this age difference may be reflected in the current results. Such differences in population and medication suggest that deprescribing activities may also be different within the general practice setting. Previous research has also suggested pharmacists as leaders of the deprescribing process; however, when focusing on general practice, GPs were overwhelmingly responsible with other healthcare professionals in supporting roles. GPs may be logical leaders for deprescribing, although they may require support from others.

\section{Strengths and limitations}

Both a limitation and strength, this review included protocol papers and protocol registrations. As deprescribing is only emerging in the literature, we thought it is important to see what activities are currently being used or will be used in general practice. Protocol papers and registrations are required to describe the intended intervention rather than the actual tested intervention therefore some activities may have been missed.

An assessment of bias was not conducted on the included studies. The most common study design included in this review was RCTs, which suggests that bias may be limited; however, most $(n=21)$ of the RCTs were described in protocol registrations or protocol papers only. As scoping reviews allow for the inclusion of a wider range of literature, the inclusion of protocols minimalised the risk of missing relevant interventions. Further, a rigorous search was conducted, allowing for a diverse set of literature to be identified in a robust and reproducible manner. Finally, previous literature has focused on studies conducted from 2002 to 2020, possibly due to 'deprescribing' only having been coined as a term in 2003. As medication discontinuation is not a recent concept, the current review may have captured some previously missed deprescribing activities.

\section{Implications for research and practice}

The addition of principle 0 and principle 6 suggests that the deprescribing process can be implemented and conducted in clinical practice cyclically, potentially creating a self-sustaining process. To preserve this 'deprescribing loop', a whole of practice approach may be needed. Certainly, the activities mapped to the additional principles indicate that initiation of deprescribing is in the hands of not only the GP but also the wider practice staff who may play an essential role in supporting the GP and patient to initiate deprescribing. In particular, practice staff will most likely be required to take on the role of identifying appropriate patients for deprescribing. Currently, research teams are heavily involved in conducting this activity; however, outside of the research setting and in clinical practice, this task will need to be assigned to nominated practice staff for this important step to be carried out. 
This scoping review has provided an overview of what activities are being used in deprescribing and operationalised them into a framework principles of deprescribing; however, guidance is still needed for how GPs might select activities for different patients and medication type. This may also assist in identifying roles for practice staff and management.

Finally, as evidence-based deprescribing principles are still lacking, the adapted principles presented in the current study should be tested and evaluated in practice. In particular, the cyclical nature of the described deprescribing process should be tested for feasibility.

\section{Conclusion}

Evidenced-based deprescribing activities and principles to guide deprescribing have yet to be combined to develop a comprehensive but easy to use guide to support GPs to deprescribe. This scoping review was the first to amalgamate deprescribing activities and pioneering deprescribing principles resulting in two additional principles. The guiding principles helped to capture the variety of deprescribing activities that currently exist in the literature and has highlighted which areas of the deprescribing process require further attention. Further, the activities included within each principle can provide guidance for GPs, practice staff and practice management teams on how they can contribute to the deprescribing process. The current findings may provide a starting point by offering a selection of deprescribing options to use in practice.

\section{Twitter Amy Coe @amy_x_coe}

Contributors All authors (AC, CK-H, SF, EM, JG) contributed to the conceptualisation and design of the study. $\mathrm{AC}$ and $\mathrm{CK}-\mathrm{H}$ performed screening. AC performed data extraction and synthesis which was reviewed by JG and CK-H. AC drafted the manuscript. JG and CK-H revised early drafts and all authors revised later drafts.

Funding AC is funded by the Rotary Club of Richmond and Kaiyu Scholarship for PhD Research in Mental Health, Australian Rotary Health.

Competing interests None declared.

Patient consent for publication Not required.

Provenance and peer review Not commissioned; externally peer reviewed.

Data availability statement All data relevant to the study are included in the article or uploaded as supplementary information. The datasets used and/or analysed during the current study are included in the article and as supplementary information.

Supplemental material This content has been supplied by the author(s). It has not been vetted by BMJ Publishing Group Limited (BMJ) and may not have been peer-reviewed. Any opinions or recommendations discussed are solely those of the author(s) and are not endorsed by BMJ. BMJ disclaims all liability and responsibility arising from any reliance placed on the content. Where the content includes any translated material, BMJ does not warrant the accuracy and reliability of the translations (including but not limited to local regulations, clinical guidelines, terminology, drug names and drug dosages), and is not responsible for any error and/or omissions arising from translation and adaptation or otherwise.

Open access This is an open access article distributed in accordance with the Creative Commons Attribution Non Commercial (CC BY-NC 4.0) license, which permits others to distribute, remix, adapt, build upon this work non-commercially, and license their derivative works on different terms, provided the original work is properly cited, appropriate credit is given, any changes made indicated, and the use is non-commercial. See: http://creativecommons.org/licenses/by-nc/4.0/.

\section{ORCID iDs}

Amy Coe http://orcid.org/0000-0003-3723-7645

Catherine Kaylor-Hughes http://orcid.org/0000-0003-3353-4108

Susan Fletcher http://orcid.org/0000-0001-6359-8650

Elizabeth Murray http://orcid.org/0000-0002-8932-3695

Jane Gunn http://orcid.org/0000-0001-8836-7525

\section{REFERENCES}

1 World Health Organization. The pursuit of responsible use of medicines: sharing and learning from country experiences, 2012. Available: http://www.who.int/medicines/publications/responsible use/en/

2 Royal Australian College of General Practitioners. The RACGP position on too much medicine, 2018. Available: https://www.racgp. org.au/advocacy/position-statements/view-all-position-statements/ clinical-and-practice-management/too-much-medicine

3 Garfinkel D, Ilhan B, Bahat G. Routine deprescribing of chronic medications to combat polypharmacy. Ther Adv Drug Saf 2015;6:212-33

4 Wallace E, McDowell R, Bennett K, et al. Impact of potentially inappropriate prescribing on adverse drug events, health related quality of life and emergency Hospital attendance in older people attending general practice: a prospective cohort study. J Gerontol A Biol Sci Med Sci 2017;72:271-7.

5 Aureen A, Slawomirski L, Klazinga N. The economics of patient safety in primary and ambulatory care: flying blind 2018;106.

6 Woodward MC. Deprescribing: achieving better health outcomes for older people through reducing medications. J Pharm Pract Res 2003;33:323-8.

7 Duncan P, Duerden M, Payne RA. Deprescribing: a primary care perspective. Eur J Hosp Pharm 2017;24:37-42.

8 Australian Institute of Health and Welfare. Australia's health 2020 data insights. Australia's health series no. 17. Cat. no. AUS 231. Canberra: AlHW, 2020.

9 Ailabouni NJ, Nishtala PS, Mangin D, et al. Challenges and enablers of deprescribing: a general practitioner perspective. PLoS One 2016;11:e0151066.

10 Reeve E, Low L-F, Shakib S, et al. Development and validation of the revised patients' attitudes towards deprescribing (rPATD) questionnaire: versions for older adults and caregivers. Drugs Aging 2016;33:913-28.

11 Steinman MA, Landefeld CS. Overcoming inertia to improve medication use and deprescribing. JAMA 2018;320:1867-9.

12 Wallis KA, Andrews A, Henderson M. Swimming against the tide: primary care physicians' views on deprescribing in everyday practice. Ann Fam Med 2017;15:341-6.

13 Doherty AJ, Boland P, Reed J, et al. Barriers and facilitators to deprescribing in primary care: a systematic review. BJGP Open 2020;4:bjgpopen20X101096 doi:10.3399/bjgpopen20X101096

14 Dills H, Shah K, Messinger-Rapport B, et al. Deprescribing medications for chronic diseases management in primary care settings: a systematic review of randomized controlled trials. J Am Med Dir Assoc 2018;19:923-35.

15 Isenor JE, Bai I, Cormier R, et al. Deprescribing interventions in primary health care mapped to the behaviour change wheel: a scoping review. Res Social Adm Pharm 2021;17:1229-41.

16 Thio SL, Nam J, van Driel ML, et al. Effects of discontinuation of chronic medication in primary care: a systematic review of deprescribing trials. Br J Gen Pract 2018;68:e663-72.

17 Dirven T, Turner C, Thio S-L, et al. Room for improvement in reporting of trials discontinuing long-term medication: a systematic review. $J$ Clin Epidemiol 2020;119:65-74.

18 Reeve E, Shakib S, Hendrix I, et al. Review of deprescribing processes and development of an evidence-based, patient-centred deprescribing process: patient-centred deprescribing process. $\mathrm{Br} \mathrm{J}$ Clin Pharmacol 2014;78:738-47.

19 Scott IA, Gray LC, Martin JH, et al. Minimizing inappropriate medications in older populations: a 10-step conceptual framework. Am J Med 2012;125:529-37.

20 Begum R, Tomlin S. Deprescribing in paediatrics. Eur J Hosp Pharm 2017;24:70:70.1-70.

21 Peters MDJ, Godfrey CM, Khalil H, et al. Guidance for conducting systematic scoping reviews. Int J Evid Based Healthc 2015;13:141-6.

22 QSR international Pty Ltd. (2020) NVivo (released in March 2020). Available: https://www.qsrinternational.com/nvivo-qualitative-dataanalysis-software/support-services/faqs

23 World Health Organization. International classification of diseases for mortality and morbidity statistics (11th revision). Available: https:// icd.who.int/browse11//-m/en. https://icd.who.int/browse11/l-m/en 
24 Walsh EK, Cussen K. 'Take ten minutes': a dedicated ten minute medication review reduces polypharmacy in the elderly. Ir Med $\mathrm{J}$ 2010;103:236-8

25 McCarthy C, Clyne B, Corrigan D, et al. Supporting prescribing in older people with multimorbidity and significant polypharmacy in primary care (SPPiRE): a cluster randomised controlled trial protocol and pilot. Implement Sci 2017;12:99.

26 Campbell AJ, Robertson MC, Gardner MM, et al. Psychotropic medication withdrawal and a home-based exercise program to prevent falls: a randomized, controlled trial. J Am Geriatr Soc 1999;47:850-3.

27 Anderson K, Freeman C, Foster M, et al. GP-led deprescribing in community-living older Australians: an exploratory controlled trial. J Am Geriatr Soc 2020;68:403-10.

28 Bashir K, King M, Ashworth M. Controlled evaluation of brief intervention by general practitioners to reduce chronic use of benzodiazepines. Br J Gen Pract 1994;44:408-12.

29 Campbell N. Reducing risk of dementia through deprescribing (R2D2). identifier NCT04270474, 2020. Available: https://clinicaltrials. gov/ct2/show/NCT04270474

30 Campins L, Serra-Prat M, Gózalo I, et al. Randomized controlled trial of an intervention to improve drug appropriateness in communitydwelling polymedicated elderly people. Fam Pract 2017;34:36-42.

31 Clyne B, Smith SM, Hughes CM, et al. Effectiveness of a multifaceted intervention for potentially inappropriate prescribing in older patients in primary care: a cluster-randomized controlled trial (OPTI-SCRIPT study). Ann Fam Med 2015;13:545-53.

32 Cossette B, Taseen R, Roy-Petit J, et al. A pharmacist-physician intervention model using a computerized alert system to reduce high-risk medication use in primary care. Eur J Clin Pharmacol 2019;75:1017-23.

33 Fried TR, Niehoff KM, Street RL, et al. Effect of the tool to reduce inappropriate medications on medication communication and deprescribing. J Am Geriatr Soc 2017;65:2265-71.

34 Giblin MJ, Clift AD. Sleep without drugs. J R Coll Gen Pract 1983;33:628-33.

35 Greiver M, Dahrouge S, O'Brien P, et al. Improving care for elderly patients living with polypharmacy: protocol for a pragmatic cluster randomized trial in community-based primary care practices in Canada. Implement Sci 2019;14:55

36 Jungo KT, Rozsnyai Z, Mantelli S, et al. 'Optimising pharmacotherapy in the multimorbid elderly in primary CAre' (OPTICA) to improve medication appropriateness: study protocol of a cluster randomised controlled trial. BMJ Open 2019;9:e031080.

37 Linsky A. Engaging patients to promote deprescribing. identifier: NCT04294901, 2020. Available: https://clinicaltrials.gov/ct2/show/ NCT04294901

38 Monteiro MA. Electronic deprescribing tool for the prevention of PIP. identifier NCT03192514, 2017. Available: https://clinicaltrials.gov/ct2/ show/NCT03192514

39 Saffar D, Saini S. Evaluation of the National randomized proton pump inhibitor De-prescribing (RaPPID) program. identifier NCT03719170, 2018. Available: https://clinicaltrials.gov/ct2/show/ NCT03719170

40 Sheppard JP, Burt J, Lown M, et al. Optimising treatment for mild systolic hypertension in the elderly (optimise): protocol for a randomised controlled non-inferiority trial. BMJ Open 2018;8:e022930.

41 Sönnichsen A, Trampisch US, Rieckert A, et al. Polypharmacy in chronic diseases-reduction of inappropriate medication and adverse drug events in older populations by electronic decision support (PRIMA-eDS): study protocol for a randomized controlled trial. Trials 2016;17:57

42 Towle I, Adams JAnovel. Pharmacist-led strategy to reduce the prescribing of benzodiazepines in Paisley. Pharm. J 2006;276:136-8.

43 Rankin A, Cadogan CA, Barry HE, et al. An external pilot cluster randomised controlled trial of a theory-based intervention to improve appropriate polypharmacy in older people in primary care (PolyPrime): study protocol. Pilot Feasibility Stud 2021;7:77.

44 Cormack MA, Sweeney KG, Hughes-Jones H, et al. Evaluation of an easy, cost-effective strategy for cutting benzodiazepine use in general practice. Br J Gen Pract 1994;44:5-8.

45 Gorgels WJMJ, Oude Voshaar RC, Mol AJJ, et al. Discontinuation of long-term benzodiazepine use by sending a letter to users in family practice: a prospective controlled intervention study. Drug Alcohol Depend 2005;78:49-56.

46 Jäger C, Freund T, Steinhäuser J, et al. Impact of a tailored program on the implementation of evidence-based recommendations for multimorbid patients with polypharmacy in primary care practicesresults of a cluster-randomized controlled trial. Implement Sci 2017;12:8.
47 Heather N, Bowie A, Ashton $\mathrm{H}$, et al. Randomised controlled trial of two brief interventions against long-term benzodiazepine use: outcome of intervention. Addict Res Theory 2004;12:141-54.

48 Johnson CF, Macdonald HJ, Atkinson P, et al. Reviewing longterm antidepressants can reduce drug burden: a prospective observational cohort study. Br J Gen Pract 2012;62:e773-9.

49 Fournier J-P, Nguyen J. Efficacy of a multi-faceted intervention to deprescribe proton pump inhibitors (PPI) in primary care: a population-based, pragmatic, cluster-randomized controlled trial. identifier: NCT04255823, 2020. Available: https://clinicaltrials.gov/ ct2/show/NCT04255823

50 Walsh K, Kwan D, Marr P, et al. Deprescribing in a family health team: a study of chronic proton pump inhibitor use. J Prim Health Care 2016;8:164

51 Wentink C, Huijbers MJ, Lucassen P, et al. Discontinuation of antidepressant medication in primary care supported by monitoring plus mindfulness-based cognitive therapy versus monitoring alone: design and protocol of a cluster randomized controlled trial. BMC Fam Pract 2019;20:105

52 Murie J, Allen J, Simmonds R, et al. Glad you brought it up: a patient-centred programme to reduce proton-pump inhibitor prescribing in general medical practice. Qual Prim Care 2012;20:141-8.

53 Kuyken W, Hayes R, Barrett B, et al. The effectiveness and costeffectiveness of mindfulness-based cognitive therapy compared with maintenance antidepressant treatment in the prevention of depressive relapse/recurrence: results of a randomised controlled trial (the PREVENT study). Health Technol Assess 2015;19:1-124.

54 Kendrick. Trial of Internet and telephone support to people coming off long-term antidepressants, 2019.

55 Luymes $\mathrm{CH}$, Poortvliet RKE, van Geloven N, et al. Deprescribing preventive cardiovascular medication in patients with predicted low cardiovascular disease risk in general practice - the ECSTATIC study: a cluster randomised non-inferiority trial. BMC Med 2018;16:5.

56 Mangin D. Maintenance antidepressants versus treatment cessation in the prevention of depression recurrence, 2008.

57 Mercier-Guyon C, Chabannes JP, Saviuc P. The role of captodiamine in the withdrawal from long-term benzodiazepine treatment. Curr Med Res Opin 2004:20:1347-55.

58 Muskens E, Eveleigh R, Lucassen P, et al. Prescribing antidepressants appropriately (PANDA): a cluster randomized controlled trial in primary care. BMC Fam Pract 2013;14:6.

59 Voshaar RCO, Gorgels WJMJ, Mol AJJ, et al. Tapering off long-term benzodiazepine use with or without group cognitive-behavioural therapy: three-condition, randomised controlled trial. Br J Psychiatry 2003;182:498-504.

60 D Sullivan M, Boudreau D, Ichikawa L, et al. Primary care opioid taper plans are associated with sustained opioid dose reduction. J Gen Intern Med 2020;35:687-95.

61 Vicens C, Fiol F, Llobera J, et al. Withdrawal from long-term benzodiazepine use: randomised trial in family practice. $\mathrm{Br} J \mathrm{Gen}$ Pract 2006;56:958-63.

62 Vicens C, Sempere E, Bejarano F, et al. Efficacy of two interventions on the discontinuation of benzodiazepines in long-term users: 36-month follow-up of a cluster randomised trial in primary care. $\mathrm{Br} J$ Gen Pract 2016;66:e85-91.

63 Miller J. Evaluating chronic pain self-management support With an opioid de-prescribing intervention, 2019. Available: https:// clinicaltrials.gov/ct2/show/NCT03890263

64 Zitman FG, Couvée JE. Chronic benzodiazepine use in general practice patients with depression: an evaluation of controlled treatment and taper-off: report on behalf of the Dutch Chronic Benzodiazepine Working Group. Br J Psychiatry J Ment Sci 2001;178:317-24.

65 Magin P. Evaluation of a multi-modal educational package for GP registrars in reviewing older patients' medication regimens and deprescribing inappropriate medications. Australian New Zealand Clinical Trials Registry (ANZCTR), 2018. Available: https://www. anzctr.org.au/Trial/Registration/TrialReview.aspx?id=374562

66 Bayliss EA, Shetterly SM, Drace ML, et al. The OPTIMIZE patientand family-centered, primary care-based deprescribing intervention for older adults with dementia or mild cognitive impairment and multiple chronic conditions: study protocol for a pragmatic cluster randomized controlled trial. Trials 2020;21:542.

67 Krol N, Wensing M, Haaijer-Ruskamp F, et al. Patient-directed strategy to reduce prescribing for patients with dyspepsia in general practice: a randomized trial. Aliment Pharmacol Ther 2004:19:917-22

68 Vejar MV, Makic MBF, Kotthoff-Burrell E. Medication management for elderly patients in an academic primary care setting: a quality improvement project. J Am Assoc Nurse Pract 2015;27:72-8. 
69 Llor C, Moragas A, Bayona C, et al. The STOP-AB trial protocol: efficacy and safety of discontinuing patient antibiotic treatment when physicians no longer consider it necessary. BMJ Open 2017;7:e015814.

70 Fernández-Liz E, Tristante PV, Martínez AA, et al. Effectiveness of an intervention to optimise the use of mirabegron for overactive bladder: a quasi-experimental study in primary care. $\mathrm{Br} J$ Gen Pract 2018;68:e852-9.

71 Holliday S, Hayes C, Dunlop A, et al. Protecting pain patients. the evaluation of a chronic pain educational intervention. Pain Med 2017;18:2306-15.
72 Magin P. Evaluation of a multi-modal educational package for GP registrars in improving guideline compliance for prescription of benzodiazepines and related drugs in general practice. ACTRN12618000824268, 2018. Available: https://www.anzctr.org.au/ Trial/Registration/TrialReview.aspx?id=375066

73 Vicens C, Leiva A, Bejarano F, et al. Intervention to reduce benzodiazepine prescriptions in primary care, study protocol of a hybrid type 1 cluster randomised controlled trial: the BENZORED study. BMJ Open 2019;9:e022046.

74 Ivers N, Jamtvedt G, Flottorp S, et al. Audit and feedback: effects on professional practice and healthcare outcomes. Cochrane Database Syst Rev 2012:CD000259. 